\title{
Contributo para o estudo da satisfação com o trabalho dos colaboradores da empresa dos Transportes Urbanos de Braga
}

\section{Contribution to the study of job satisfaction with the work of the employees of Transportes Urbanos de Braga}

\author{
Ana Peixoto*, Fátima Lobo** \& Augusta Macieira*** \\ *Aluna do Mestrado em Psicologia do Trabalho e das Organizações da Universidade Católica/ Braga, **Centro de Estudos \\ Filosóficos e Humanísticos da Universidade Católica/ Braga, ***Transportes Urbanos de Braga (TUB). PEst-OE/FIL/UI0683/2015
}

\begin{abstract}
Resumo
Esta investigação tem por finalidade contribuir para a satisfação e qualidade de vida dos colaboradores da empresa de transporte público, Transportes Urbanos de Braga/Empresa Municipal (TUB/EM), de forma a funcionar como um elemento interveniente do sucesso individual e organizacional. Os trabalhadores atualmente são considerados colaboradores ou parceiros, e não mera mão-de-obra, visto que a sua satisfação pode influenciar os processos organizacionais, a qualidade do produto e a produtividade. Com efeito, torna-se essencial quer para o trabalhador, quer para a própria organização a avaliação da satisfação no trabalho, pois permite à organização identificar a necessidade de implementar estratégias que melhorem a qualidade de vida no trabalho e a qualidade da organização. Assim, este projeto de diagnóstico à satisfação com o trabalho, foi realizado através de um questionário construído para o efeito, composto por 54 questões abertas e fechadas, e a população alvo foi o universo de colaboradores dos TUB. A amostra probabilística de 201 sujeitos, maioritariamente masculina $(89,1 \%)$, com escolaridade obrigatória $(35,8 \%)$ e ensino secundário $(32,3 \%)$. Os resultados indicam que os colaboradores se encontram relativamente satisfeitos, sendo que estão mais satisfeitos com a dimensão intrínseca da satisfação no trabalho, isto é com o próprio trabalho e o seu conteúdo, revelando-se menos satisfeitos com os aspetos extrínsecos, nomeadamente com o ambiente físico e oportunidades de formação e atualização oferecidas pela empresa.
\end{abstract}

Palavras-chave: satisfação com o trabalho, colaboradores, Transportes Urbanos de Braga.

\footnotetext{
Abstract

This research aims to contribute to the satisfaction and quality of life the public transport company employees, Transportes Urbanos de Braga/Empresa Municipal (TUB/EM), in order to function as an intervening element of the individual and organizational success. The workers are currently considered employees or partners, not mere hand labor, since their satisfaction can influence organizational processes, product quality
}

and productivity. Indeed, it is essential both for the worker and for the own organization the evaluation of job satisfaction because it allows the organization to identify the need to implement strategies that improve the quality of life at work and the organization's quality. Thus, this diagnostic project to job satisfaction was conducted through a questionnaire constructed for this purpose, it consists of 54 open and closed questions, and the target population was the universe of employees of TUB. A probabilistic sample of 201 subjects, mostly male $(89,1 \%)$, with compulsory schooling $(35,8 \%)$ and secondary education $(32,3 \%)$.

The results indicate that employees are relatively satisfied, and are more satisfied with the intrinsic dimension of job satisfaction, that is with their own work and its contents, revealing less satisfied with the extrinsic aspects, particularly the environment physical and opportunities for training and updating offered by the company.

Keywords: job satisfaction, employees, Transportes Urbanos de Braga.

\section{Introdução}

Este trabalho visa contribuir para o estudo da satisfação com o trabalho dos colaboradores da empresa de transporte urbano de passageiros da cidade de Braga, TUB. Os TUB, instituídos em 1882, atuam no setor do transporte urbano de passageiros, operando no município de Braga. Com mais de um século de história atravessaram várias fases e transformações até aos dias de hoje. Ao longo dos últimos anos, a TUB/EM tem demonstrado um crescimento forte e sustentado o que permite considera-la como uma empresa sólida e dinâmica, que tem procurado inverter o paradigma da mobilidade em Braga (Transportes Urbanos de Braga, 2014).

A melhor compreensão da satisfação com o trabalho dos colaboradores, objeto de estudo deste trabalho, reveste-se de grande importância. Ao longo deste trabalho de investigação procurar-se-á atingir os seguintes objetivos: (1) conhecer o grau de satisfação dos colaboradores; (2) em que medida os colaboradores estão satisfeitos com a organização, com a supervisão, com as relações sociais no posto de trabalho, com o ambiente físico e com a tarefa; (3) conhecer a relação 
entre as variáveis sociodemográficas e o grau de satisfação; e (4) avaliar e analisar a existência de riscos psicossociais e a relação trabalho-família.

\section{Da definição à avaliação da satisfação com o trabalho}

O trabalho passou a ser central na vida das pessoas, e a satisfação com ele adquirida tem sido considerada um fator relevante associado à saúde mental. $\mathrm{O}$ interesse científico sobre a satisfação no trabalho e a sua influência na qualidade de vida dentro e fora das organizações teve origem nos anos 30 (Lawler, 1983, cit. in Carlotto \& Câmara, 2008). Contudo, segundo Pocinho e Garcia (2008), a história do estudo da satisfação no trabalho é relativa ao ano de 1912 com os estudos de Taylor sobre o efeito do ambiente de trabalho, nomeadamente a fadiga e o salário, no desempenho do trabalhador, a sua satisfação e produtividade.

A satisfação dos trabalhadores incita as organizações a mobilizar esforços. Aliás, os trabalhadores atualmente são considerados colaboradores ou parceiros, e não como mera mão-de-obra, atendendo que a sua satisfação pode influenciar os processos organizacionais, a qualidade do produto e a produtividade (Hernandez \& Melo, 2003).

De acordo com Jewell, Beavers, Kirby e Flowers (2001), a satisfação no trabalho e as suas variáveis determinantes (produtividade, desempenho, absentismo e abandono laboral, entre outros), são das dimensões mais investigadas nos últimos anos no campo da Psicologia Organizacional, constituindo-se mesmo como o constructo mais estudado pela comunidade científica. Este interesse pela variável deve-se ao seu efeito na vida das organizações, quer ao nível específico (no domínio do trabalhador), quer no âmbito da atividade global da própria organização (Hirschfeld, 2000).

A satisfação com o trabalho é uma dimensão complexa e de difícil definição, por se tratar de um estado subjetivo, podendo variar de sujeito para sujeito, segundo diferentes circunstâncias, e ao longo do tempo para a mesma pessoa. É compreendida como um fator preditor nos aspetos da produtividade e da realização dos trabalhadores (Carlotto \& Câmara, 2008).

A satisfação no trabalho é abordada como um conjunto de sentimentos favoráveis a partir dos quais os trabalhadores compreendem o seu trabalho. Para Locke (1976), é o resultado da perceção de que o próprio trabalho reflete os valores laborais mais importantes para o trabalhador, na medida em que estes são congruentes com as suas necessidades. A satisfação e a insatisfação no trabalho são respostas emocionais com uma diversidade de ações alternativas que podem funcionar como respostas no campo psicológico ou comportamental. De acordo com este autor os componentes motivadores da satisfação laboral estão relacionados com o seu conteúdo, as possibilidades de promoção, o reconhecimento, as condições e o ambiente de trabalho, as relações com colegas e subordinados, as caraterísticas da supervisão e da gestão, e as políticas e competências da empresa (Locke, 1976).

Mais recentemente, a satisfação no trabalho é abordada na perspetiva de Tamayo (2000, cit. in Carlotto \& Câmara, 2008), como uma variável multifatorial, incluindo aspetos como: salário, colegas, supervisão, oportunidades para promoção, benefícios, condições de trabalho, progressão na carreira, estabilidade no trabalho, desenvolvimento pessoal e quantidade de trabalho.

A constituição deste constructo tem por base três vertentes: individual, situação laboral e caraterísticas do trabalho. A individual engloba as necessidades pessoais, os valores e os traços da personalidade; a situação laboral reflete frequentemente as comparações sociais que os indivíduos fazem com outros trabalhadores, as características de trabalhadores anteriores e os grupos de referencia; e por último as caraterísticas do trabalho, como retribuição, condições de trabalho, supervisão, colegas, conteúdo do trabalho, segurança no emprego e oportunidades de progresso, entre outras (Ferrer \& Rodríguez, 2009).

As causas da satisfação podem ser pessoais ou organizacionais. As primeiras dividem-se em causas demográficas e diferenças individuais, assim a idade e género, são os fatores demográficos com mais elevada correlação. Por sua vez, tendo em conta as causas organizacionais, o principal elemento que contribui para a satisfação nas organizações é o salário, por permitir a resolução de necessidades individuais e também o estatuto e reconhecimento social. A satisfação tem importância ao nível da compreensão do comportamento humano em termos organizacionais. $\mathrm{O}$ trabalho em si mesmo e as oportunidades de promoção são outros dois fatores de satisfação. A promoção é abordada como causa de satisfação por refletir ganhos em termos remunerativos, estatuto e prestígio; as caraterísticas de supervisão, as características dos colegas e as condições físicas de trabalho, são outros elementos que favorecem a satisfação dos colaboradores nas organizações (Cardoso, Cunha, Cunha, \& Rego, 2003).

A satisfação laboral correlaciona-se com outras variáveis laborais, como motivação, dedicação ao trabalho, comportamento de cidadania organizacional, absentismo, rotatividade de trabalhadores, stresse e produtividade laboral. Nos últimos anos, o interesse focaliza a análise da relação entre a satisfação no trabalho e a saúde e o bem-estar dos trabalhadores. A insatisfação laboral pode ser considerada como um fator preditor de doença grave, podendo mesmo causar a morte. Algumas investigações demonstraram que trabalhadores insatisfeitos manifestavam mais sintomas físicos, como problemas de estômago e sono, que os seus colegas. Da mesma forma, revelaram que a insatisfação se vincula com as emoções negativas no trabalho, como a angústia e a depressão. Os estados afetivos podem ser considerados como indicadores de bem-estar emocional no trabalho (Ferrer \& Rodríguez, 2009).

A satisfação no trabalho reflete-se no trabalhador e simultaneamente no funcionamento da organização. 
Com efeito, torna-se essencial quer para o trabalhador, quer para a própria organização a avaliação da satisfação no trabalho, por se tratar de um procedimento comum em organizações que zelam pelo bem-estar dos seus colaboradores, seja por fundamentos humanísticos ou de eficácia e eficiência organizacional (Saane, Sluiter, Verbeek, \& FringsDresen, 2003).

A avaliação da satisfação no trabalho permite à organização identificar a necessidade de implementar estratégias que melhorem a qualidade de vida no trabalho e a qualidade da organização (Saane et al, 2003). Um trabalhador satisfeito preocupa-se mais com a qualidade das funções que desempenha, valoriza o cliente, compromete-se mais com a organização, sendo o seu trabalho mais produtivo. Por sua vez, uma organização com trabalhadores satisfeitos melhora a qualidade do seu produto ou serviço, aumenta a satisfação do seu cliente, reduz os custos com o abandono laboral e necessário processo de recrutamento e formação de novos trabalhadores, aumentando a produtividade (Ngo, 2009, cit. in Ferreira, Fernandes, Haase, \& Santos, 2009). É nesta interação mútua que a satisfação no trabalho pode funcionar como um elemento interveniente do sucesso individual e organizacional (Ferreira et al, 2009).

\section{Metodologia}

\section{Participantes}

Do universo de colaboradores da empresa TUB, participaram neste estudo 179 colaboradores e 22 colaboradoras (constituindo uma amostra total de 201 participantes) variando a faixa etária entre os 25 e os 64 anos $(\mathrm{M}=46,50, \mathrm{DP}=9,19)$. Quanto ao estado civil, verificou-se que $77,6 \%$ são casados e $13,9 \%$ são solteiros, constituindo estes dois estados a maioria da amostra. Relativamente ao número de filhos, verificouse que varia entre 0 e 5 , sendo que a maioria tem um e dois filhos, $32,3 \%$ e $45,8 \%$ respetivamente.

A fim de garantir uma amostra heterogénea, incluíram-se participantes de diferentes departamentos e seções: 32 da área da manutenção, 8 da área das vendas, 121 agentes únicos e 40 colaboradores que exercem outras funções. No que diz respeito ao tipo de horário praticado, 71 têm horário fixo, 33 fazem o $1^{\circ}$ turno, 41 o $2^{\circ}$ turno, 31 as 3 etapas, 11 o $1^{\circ}$ e $2^{\circ}$ turno, 2 o $1^{\circ}$ turno e 3 etapas, e 11 fazem o $1^{\circ}, 2^{\circ}$ turno e 3 etapas.

Entre os participantes, verificou-se alguma variedade quanto ao local de residência, incluindo 169 de Braga, 10 de Vila Verde, 4 de Amares, 3 de Barcelos e Vieira do Minho, 2 de Guimarães e Póvoa de Lanhoso, e 1 de Esposende, Montalegre e Famalicão.

Quanto ao nível educacional, verificou-se que 72 colaboradores possuem habilitações literárias ao nível da escolaridade obrigatória, 65 do ensino secundário, 15 licenciados, 3 mestrado e 44 com outro nível de formação.

\section{Instrumento}

A realização do diagnóstico à satisfação dos colaboradores consistiu, numa primeira fase, na construção do questionário de avaliação. O questionário é constituído por um conjunto de questões sociodemográficas e um outro conjunto que avalia a satisfação com a atividade da organização, com a supervisão, com as relações sociais no posto de trabalho, com a satisfação pessoal e o ambiente físico. Foram ainda colocadas três questões abertas, duas para analisar a utilização dos serviços da empresa por parte dos colaboradores e dos respetivos agregados familiares, e uma outra no sentido de se perceber o que a empresa poderá fazer para aumentar a satisfação dos seus colaboradores. $\mathrm{O}$ questionário tem uma escala de resposta de 5 pontos em que 1 significa totalmente insatisfeito, 2 insatisfeito, 3 satisfeito, 4 muito satisfeito e 5 totalmente satisfeito.

Este questionário foi ainda utilizado para a realização de uma segunda avaliação, com o objetivo de apurar se as caraterísticas inerentes às condições e organização do trabalho afetam a saúde dos colaboradores, através de processos psicológicos e fisiológicos, isto é a exposição a possíveis riscos psicossociais (Da Silva, 2012). As dimensões de avaliação dizem respeito à qualidade do sono, ao stresse, ao burnout, às exigências emocionais, bem como à relação trabalho-família. Estas dimensões são avaliadas numa escala tipo Likert de 5 pontos ( 1 - nunca/quase nunca, 2 - raramente, 3 às vezes, 4 - frequentemente e 5 - sempre), sendo que a dimensão relação trabalho-família foi avaliada através de duas questões abertas, designadamente se o trabalho interfere com a vida familiar $\mathrm{e}$ se as responsabilidades familiares interferem com o trabalho.

\section{Procedimento}

Após ter sido aprovado e autorizado pela administração da organização, a segunda fase da avaliação compreendeu a aplicação e recolha dos questionários, a qual decorreu entre Dezembro de 2014 e Março de 2015. A aplicação realizou-se por departamentos, começando pela área administrativa e demais seções, seguindo-se a área da manutenção, os funcionários dos postos de venda, e por fim os agentes únicos, a percentagem mais significativa de colaboradores da empresa. A aplicação dos questionários foi adaptada consoante os horários e disponibilidade dos colaboradores, particularmente no caso dos agentes únicos.

Uma vez recolhidos todos os questionários procedeuse à sua inserção no programa de tratamento estatístico Statistical Package for Social Sciences (SPSS) versão 20. Realizou-se, inicialmente, a análise de conteúdo das seguintes questões: "Utiliza os serviços dos TUB?", "E o seu agregado familiar?", “O que gostaria de receber da empresa para aumentar a sua satisfação?", "O seu trabalho interfere com a sua vida familiar?", "As suas responsabilidades familiares interferem com o seu trabalho?", e por último as "sugestões". Posteriormente foi realizada a análise dos dados quantitativos, centrando-se em análises gerais 
sobre a satisfação profissional, nomeadamente estatísticas de caráter descritivo.

\section{Resultados}

Em análise à pergunta "Utiliza os serviços dos TUB?", verificou-se que 125 inquiridos utilizam e 76 não utilizam os serviços. Quanto à pergunta "E o seu agregado familiar?", 95 responderam que sim e 96 responderam que não utilizam.

Procurou-se perceber o que os colaboradores gostariam de receber da empresa para aumentar a sua satisfação e verificou-se que a opção com maior registo de respostas foi o ginásio $(n=93)$, depois referiram outras sugestões diversas $(n=40)$, um centro de atividades para ocupação de tempos livres dos filhos (n $=39)$, e por último, um refeitório $(\mathrm{n}=13)$.

Através dos resultados dos diversos itens sobre a satisfação com a atividade da organização, foi possível constatar que os colaboradores se encontram relativamente satisfeitos apresentando uma média que varia entre os 3,10 e os 3,69 num total possível de 5 . Tendo em conta os valores máximos para cada item, verifica-se que os colaboradores se mostram mais satisfeitos com o empenho e os objetivos da empresa, sendo que este último item foi o único que não teve respostas com a cotação 1 (totalmente insatisfeito). Por sua vez, o item que revelou valores mais baixos de satisfação foi com as oportunidades de formação e atualização oferecidas pela empresa $(\mathrm{M}=2,94$, $\mathrm{DP}=$ $1,15)$.

Quanto à satisfação com a supervisão, constatou-se que os participantes se manifestam moderadamente satisfeitos, variando a média entre os 3,73 e os 3,90, sendo que estão mais satisfeitos com as relações com os seus superiores hierárquicos diretos $(\mathrm{M}=3,90$, $\mathrm{DP}=.964)$.

$\mathrm{Na}$ dimensão relativa à satisfação com as relações sociais no posto de trabalho, a média varia entre os 3,55 e os 3,79 , o que revela que os colaboradores se encontram relativamente satisfeitos com as suas relações sociais, estando mais satisfeitos com o ambiente de trabalho $\mathrm{e}$ as relações pessoais e profissionais no posto de trabalho.

A média dos resultados dos itens sobre a satisfação pessoal varia entre 3,21 e 4,35, mostrando que os colaboradores se mostram muito satisfeitos com a dedicação e empenho na concretização das funções; as funções que executam; e a qualidade do trabalho desenvolvido. $\mathrm{O}$ item que registou o valor mais baixo foi a possibilidade de participar na tomada de decisões $(\mathrm{M}=3,21, \mathrm{DP}=1,11)$.

Ao nível dos itens relativos à satisfação com o ambiente físico, verificou-se que os colaboradores se encontram pouco satisfeitos, sendo que o item sobre a limpeza, higiene e segurança do local de trabalho, apresentou um dos níveis médios mais baixos nesta $\operatorname{amostra}(\mathrm{M}=2,97, \mathrm{DP}=1,02)$.

Para a apresentação dos resultados da dimensão bemestar, os itens foram divididos em dois grupos, aqueles cujo valor mais alto corresponde ao pior valor e aqueles em que o valor mais baixo corresponde ao melhor valor. Na amostra o ponto de corte para a presença de valores críticos é 3,66 e o ponto de corte para os melhores valores é 2,33. Assim, o item com pior pontuação, foi $\mathrm{o}$ trabalho emocionalmente exigente, 3,45. Os restantes itens apresentaram uma boa pontuação: problemas em dormir $(1,94)$, burnout $(2,11)$, e stresse $(2,14)$.

Em relação à questão sobre se o trabalho interfere na vida familiar, 136 dos colaboradores responderam que "não" e 60 que "sim". Sobre se as responsabilidades familiares interferem com o trabalho, a maioria dos colaboradores, 186, mencionou que não, e apenas 10 referiram que sim.

\section{Análise e Discussão}

De acordo com Jewell, Beavers, Kirby e Flowers (2001), a satisfação no trabalho e as suas variáveis determinantes (produtividade, desempenho, absentismo e abandono laboral, entre outros), são as dimensões mais investigadas nos últimos anos no campo da Psicologia Organizacional, constituindo-se mesmo como o constructo mais estudado pela comunidade científica. Este interesse pela variável deve-se ao seu efeito na vida das organizações, quer ao nível específico (no domínio do trabalhador), quer no âmbito da atividade global da própria organização (Hirschfeld, 2000).

É compreendida como um fator preditor nos aspetos da produtividade e da realização dos trabalhadores (Carlotto \& Câmara, 2008). Na interação mútua entre trabalhador e organização, a satisfação no trabalho pode funcionar como um elemento interveniente do sucesso individual e organizacional (Ferreira et al, 2009).

Os resultados obtidos neste estudo apontam para uma relativa satisfação dos 201 colaboradores, quer pela análise das respostas às questões, quer pela análise das médias obtidas.

$\mathrm{Na}$ análise das dimensões da satisfação, verificou-se que os participantes se manifestam mais satisfeitos com a dimensão intrínseca da satisfação no trabalho, isto é com o próprio trabalho e o seu conteúdo, revelando-se menos satisfeitos com os aspetos extrínsecos, nomeadamente com o ambiente físico $\mathrm{e}$ as oportunidades de formação e atualização oferecidas pela empresa.

Da mesma forma, outros estudos sobre satisfação no trabalho obtiveram resultados superiores na dimensão intrínseca. O estudo de Seco (2000), revelou que trabalhadores de diversos países (Bélgica, Inglaterra, Israel e EUA) indicaram a natureza do trabalho como o aspeto mais importante. No mesmo sentido, em Portugal existem dados que apontam para uma valorização da dimensão intrínseca (Seco, 2000).

Nesta amostra, os dados são reforçados pela análise das médias obtidas ao nível dos itens da escala da satisfação, detetando-se maior satisfação com o próprio trabalho e o seu conteúdo e satisfação mais baixa no que diz respeito às oportunidades de formação oferecidas pela organização. Um aspeto a realçar relaciona-se com $\mathrm{o}$ facto dos participantes se 
mostrarem muito satisfeitos com a dimensão extrínseca da supervisão.

Altos graus de satisfação estão associados, entre outros fatores, com as oportunidades de formação e progressão na carreira. Em Portugal, a implementação de planos de formação por parte das organizações não tem sido um procedimento comum, à exceção da formação informal que tem como objetivo a preparação dos trabalhadores para o desempenho das suas funções (Parente, 1996). Um estudo realizado pela Fundação Europeia para a Melhoria das Condições de Vida e de Trabalho, sobre as condições de trabalho na União Europeia, revelou que apenas 13\% dos trabalhadores portugueses participaram em ações de formação, enquanto a média europeia é de 38\% (Graça, 1999).

Os resultados dos itens da dimensão bem-estar são interpretados mediante $o$ valor mais alto que corresponde ao pior valor e o valor mais baixo que corresponde ao melhor valor, representando o impacto para a saúde da exposição a determinado fator. $\mathrm{Na}$ amostra, os itens da dimensão estão numa situação favorável para a saúde, à exceção do item relativo ao trabalho ser emocionalmente exigente, que por apresentar o valor mais elevado $(3,45)$ representa uma situação intermédia de risco para a saúde.

Geralmente, os efeitos do trabalho na saúde não se manifestam de forma linear e imediata, e embora estejam presentes em todo o ciclo de vida do indivíduo, a sua expressão é frequentemente singular e insidiosa (Gollac \& Volkoff, 2000, cit. in Ribeiro, 2008). As condições de trabalho, de facto, são vividas de forma diferente e as suas consequências dependem do percurso profissional e do contexto de trabalho, assim como da perceção que cada um tem segundo as suas capacidades físicas, psicológicas e sociais (Duarte \& Lacomblez, 2006).

Os resultados da análise qualitativa, neste estudo, revelam que os motivos acerca da utilização dos serviços são paralelos para as diferentes funções. Os principais motivos apontados para a utilização dos serviços dizem respeito ao facto de ser mais económico e de ser o meio de transporte para o trabalho. Por sua vez, quando não utilizam evidenciam como causa a utilização do transporte próprio.

Quanto à utilização dos serviços por parte do agregado familiar, destaca-se a utilização como meio de transporte para o trabalho, mas também ao centro da cidade e para a escola. Relativamente, à não utilização dos serviços mais uma vez justifica-se pela preferência pelo transporte próprio.

Apesar de serem conhecidas as vantagens do transporte público e os efeitos do uso intensivo do transporte individual, caraterísticas como a flexibilidade, permanente disponibilidade, privacidade, comodidade e conforto que fazem do automóvel praticamente uma extensão natural da habitação, continuam a privilegiar claramente a sua utilização (Da Costa, 2008).

$\mathrm{Na}$ relação trabalho-família, é de realçar que são os agentes únicos os colaboradores mais afetados por este conflito, uma vez que refletem o número mais significativo de respostas afirmativas a esta questão. Como causas a este conflito destacam, devido ao trabalho por turnos, os horários de trabalho e o pouco tempo para a família.

À medida que a economia se direciona para a indústria e para a prestação de serviços, o recurso ao trabalho por turnos e noturno surge como uma necessidade intrínseca ao processo produtivo, sendo por isso cada vez mais frequente. Um problema do trabalho por turnos é o horário que para além de afetar a qualidade do sono, limita a vida familiar (Ribeiro, 2008).

O trabalho por turnos implica, assim, que as horas disponíveis para as diversas atividades diárias, de lazer e da vida familiar e social, fique comprometido. Este impacto é mais negativo quando os dias de trabalho são ao fim-de-semana, uma vez que a sociedade é uma "sociedade da tarde", a qual atribui mais importância às horas do fim da tarde e do fim-de-semana para a vida familiar e social (Gadbois, 1998, cit. in Ribeiro, 2008).

Para aumentar a satisfação, os colaboradores destacaram que gostariam de receber da empresa um ginásio. Os benefícios sociais disponibilizados aos colaboradores são cada vez mais valorizados pelas empresas, entre os quais os ginásios têm preferência. Entre os empresários e responsáveis pela gestão de recursos humanos estabelece-se a noção de que este benefício social contribui para a saúde e qualidade de vida dos seus colaboradores. A promoção da atividade física regular dos colaboradores pode levar a uma diminuição do nível de absentismo nas empresas, melhorando o ambiente de trabalho e consequentemente a produtividade (Gomes, 2013).

Através da área de sugestões os colaboradores salientaram diversas necessidades, sendo as mais expressivas as melhorias das instalações e condições de trabalho, mais oportunidades de formação e de atualização, e aumento nos salários.

Estes dados são reforçados pela análise estatística dos dados, quando se verifica menor satisfação no que diz respeito às oportunidades de formação oferecidas pela empresa e ao ambiente físico.

\section{Considerações finais}

Através dos resultados obtidos neste estudo, verificase que os resultados de ambas as metodologias utilizadas neste estudo, análise quantitativa e análise qualitativa, se reforçam e complementam. O método qualitativo possibilita uma análise para a projeção da empresa em termos de representações ideais e expectativas dos colaboradores, por sua vez o quantitativo permite uma análise sobre o que está a ser feito, refletindo as representações reais dos colaboradores.

Seria interessante em próximos trabalhos, investigar os resultados superiores de satisfação encontrados nas dimensões mais intrínsecas relacionadas com o próprio trabalho e o seu conteúdo. De facto, o trabalho assume uma importância central na vida do ser humano, estruturando a sua relação com o mundo e as suas relações sociais, e reforçando as sociedades de forma a 
possibilitar que os indivíduos sejam parte ativa de uma relação social e se integrem na comunidade (Araújo, 2009).

Outro aspeto a explorar, refere-se a um dos aspetos extrínsecos que também revelou resultados superiores, a satisfação com a supervisão. A supervisão é considerada como um dos fatores que mais influência tem sobre a satisfação, sendo por isso um fenómeno cada vez mais estudado. De acordo com Watson (2009), perceber de que forma os comportamentos de liderança dos superiores hierárquicos influenciam a satisfação dos colaboradores, permitirá desenvolver comportamentos e criar um clima de trabalho que beneficie as necessidades dos trabalhadores.

Neste sentido, seria interessante em investigações futuras estudar outros indicadores que poderão estar associados às diferenças de satisfação no trabalho, como por exemplo, o tipo de vínculo laboral, o tempo de permanência na organização, caraterísticas da supervisão, clima de trabalho, assim como explorar fatores que poderão estar associados às diferenças de horário ou idade, como por exemplo as condições físicas e psicológicas dos colaboradores, a atividade profissional por turnos, a fim de compreender associações entre as variáveis e a satisfação.

\section{Referências}

Araújo, P. (2009). “Os inempregáveis”: estudos de caso sobre os impactos psicossociais do nãoemprego em licenciados portugueses." Dissertação de mestrado não publicada. Faculdade de Psicologia e de Ciências da Educação da Universidade do Porto.

Cardoso, C., Cunha, C. R., Cunha, M. P., \& Rego, A. (2003). Manual de Comportamento Organizacional e Gestão. Lisboa: RH Editora.

Carlotto, M. S., \& Câmara, S. G. (2008). Propriedades psicométricas do Questionário de Satisfação no Trabalho (S20/23). Psico-USF, 13 (2), 203-210.

Chambel, M. J., \& Curral, L. (1995). Psicossociologia das Organizações. Lisboa: Texto Editora.

Da Costa, A. (2008). Transportes públicos. Manual do planeamento de acessibilidades e transportes. Comissão de coordenação e desenvolvimento regional do norte.

Da Silva, C. F. (2012). Copenhagen Psychosocial Questionnaire - COPSOQ - Versão Portuguesa. Aveiro: Análise Exacta.

Duarte, B. C., \& Lacomblez, M. (2006). Saúde no trabalho e discrição das relações sociais. Laboreal, 2 (2), 82-92.

Ferrer, C. M., \& Rodríguez, M. A. (2009). Actitudes hacia el trabajo: satisfacción, implicación $\mathrm{y}$ compromiso laboral. In A. R. Fernández, V. Z. Martín \& J. M. González (Eds.). Psicología del Trabajo. Ediciones Pirámide.
Ferreira, J. A., Fernandes, R., Haase, R. F., \& Santos, E. R. (2009). Minnesota Satisfaction QuestionnaireShort Form: estudo de adaptação e validação para a população portuguesa. PSYCHOLOGICA, 59, 251281.

Gomes, A. S. (2013, 30 maio). Empresas levam colaboradores ao ginásio. O Jornal Económico, 1116.

Graça, L. (1999). Qualidade e Condições de Trabalho. Consultado em Maio de 2015, http://www.ensp.unl.pt/luis.graca/textos6.html

Hernandéz, J. A., \& Melo, F. M. (2003). O clima organizacional e a satisfação dos funcionários de um Centro Médico Integrado. rPOT, 3 (1), 11-26.

Hirschfeld, R. (2000). Does revising the intrinsic and extrinsic subscales of the Minnesota Satisfaction Questionnaire short form make a difference? Educational Psychology Measure, 60, 255-270.

Jewell, L., Beavers, K., Kirby, B. M., \& Flowers, J. (2001). Relationships between levels of job satisfaction expressed by North Carolina vocational agriculture teachers and their perceptions toward the agricultural education teaching profession. Journal of Agricultural Education, 31 (1), 52-57.

Locke, E. (1976). The nature and causes of job satisfaction. In M. Dunnette (Ed.) Handbook of Industrial and Organizational Psychology. Chicago: Rand McNally.

Parente, C. (1996). As empresas como espaço de formação. Consultado em Maio de 2015, http://ler.letras.up.pt/uploads/ficheiros/1394.pdf

Pocinho, M. D., \& Garcia, J. C. (2008). Impacto Psicosocial de la Tecnología de Información y Comunicación (TIC): Tecnoestrés, Daños Físicos y Satisfacción Laboral. Acta Colombiana de Psicología, 11 (2), 127-139.

Ribeiro, M. E. (2008). Consequências do trabalho por turnos e nocturno em profissionais de enfermagem. Dissertação de mestrado. Faculdade de Ciências Humanas e Sociais, Universidade Fernando Pessoa.

Saane, N., Sluiter, J. K., Verbeek, J. H., \& FringsDresen, M. H. (2003). Reliability and validity of instruments measuring job satisfaction - a systematic review. Occupational Medicine, 53 (3), 190-200.

Seco, G. (2000). A satisfação na actividade docente. Tese de Doutoramento não publicada. Faculdade de Psicologia e de Ciências da Educação, Universidade de Coimbra.

Transportes Urbanos de Braga (2014). Manual de Gestão da Qualidade.

Watson, L. (2009). Leadership's influence on job satisfaction. Radiologic Technology, 80 (4), 297-308 